SSC00-II-8

\title{
Low Cost Planetary Exploration: Surrey Lunar Minisatellite and Interplanetary Platform Missions
}

\author{
Susan Jason \\ Dr. Jeff Ward \\ Alex da Silva Curiel \\ Andy Phipps \\ Luis Gomes \\ Prof. Martin Sweeting \\ Surrey Space Centre \\ University of Surrey \\ Guildford, Surrey GU2 7XH, UK \\ Tel: (44) 1483259278 Fax: (44) 1483259503 \\ Internet: m.sweeting@eim.surrey.ac.uk \\ WWW: http://www.sstl.co.uk
}

\begin{abstract}
In order to meet the growing global requirement for affordable missions beyond Low Earth Orbit, two types of platform are under design at the Surrey Space Centre. The first platform is a derivative of Surrey's UoSAT-12 minisatellite, launched in April 1999 and operating successfully in-orbit. The minisatellite has been modified to accommodate a propulsion system capable of delivering up to $1700 \mathrm{~m} / \mathrm{s}$ delta-V, enabling it to support a wide range of very low cost missions to LaGrange points, Near-Earth Objects, and the Moon. A mission to the Moon - dubbed "MoonShine" - is proposed as the first demonstration of the modified minisatellite beyond LEO. The second platform - Surrey's Interplanetary Platform - has been designed to support missions with delta-V requirements up to $3200 \mathrm{~m} / \mathrm{s}$, making it ideal for low cost missions to Mars and Venus, as well as Near Earth Objects (NEOs) and other interplanetary trajectories.

Analysis has proved mission feasibility, identifying key challenges in both missions for developing cost-effective techniques for: spacecraft propulsion; navigation; autonomous operations; and a reliable safe mode strategy. To reduce mission risk, inherently failure resistant lunar and interplanetary trajectories are under study. In order to significantly reduce cost and increase reliability, both platforms can communicate with low-cost ground stations and exploit Surrey's experience in autonomous operations. The lunar minisatellite can provide up to $70 \mathrm{~kg}$ payload margin in lunar orbit for a total mission cost US\$16-25 M. The interplanetary platform can deliver $20 \mathrm{~kg}$ of scientific payload to Mars or Venus orbit for a mission cost US\$25-50 M. Together, the platforms will enable regular flight of payloads to the Moon and interplanetary space at unprecedented low cost. This paper outlines key systems engineering issues for the proposed Lunar Minisatellite and Interplanetary Platform Missions, and describes the accommodation and performance offered to planetary payloads.
\end{abstract}




\section{Introduction}

Surrey's approach to Low Cost Satellite Engineering and Small Satellite Programme Management has been verified through the in-orbit success of 16 microsatellites, a minisatellite and a nanosatellite. Use of the Surrey Small Satellite Management and Engineering Approach has enabled rapid implementation of new techniques and advances in commercially available technologies, resulting in reliable, yet increasingly capable spacecraft at low cost. This successfully proven approach will be employed throughout all phases of the lunar and interplanetary missions.

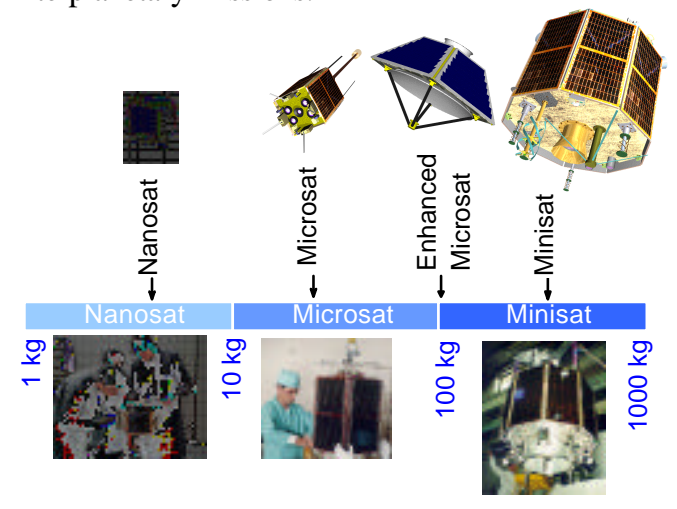

Figure 1 Spectrum of Surrey Satellite

Platforms

Surrey’s capabilities (Figure 1) range from very low cost $(<\$ 1$ $\mathrm{M})$ gravity gradient microsatellites carrying store and forward communications payloads, to the $350 \mathrm{~kg}$ UoSAT-12 minisatellite demonstrating among other features - autonomous orbit determination and control, $10 \mathrm{~m}$ GSD (panchromatic) and multispectral imaging [1]. The $50 \mathrm{~kg}$ Tsinghua-1 microsatellite built with Tsinghua University, China, provides 39 m GSD multispectral imaging and has an off-pointing capability to increase coverage and flexibility in imaging [2]. The $7 \mathrm{~kg}$ SNAP-1 nanosatellite platform is paving the way for future satellite "swarm" applications - demonstrating a range of miniaturised Surrey subsystems, from cold gas propulsion and GPS navigation, to an S-band downlink and momentum wheels for 3-axis stabilisation [3].

Surrey is also currently working on micro- and mini-satellite constellations with a variety of customers - with applications ranging from commercial agriculture and disaster monitoring [4] to global sea state monitoring [5] and LEO global messaging services.

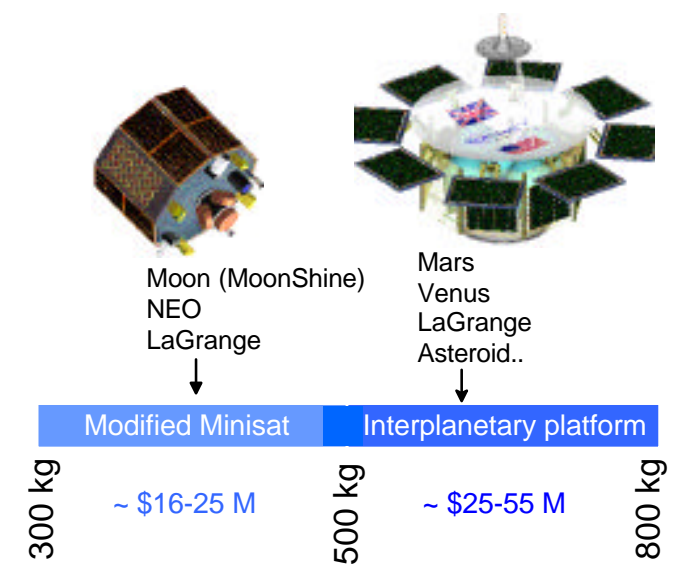

Figure 2 Surrey Planetary Satelites Spectrum

The interplanetary and lunar minisatellite platforms described in this paper build upon the experience gained through all Surrey Space Centre research, development and commercial activities. Together, the modified minisatellite and interplanetary platforms can perform a wide range of missions beyond LEO. Figure 2 shows the complementarity of the platforms in covering a wide range of mission applications over an accommodating spectrum of low-cost budgets.

\section{Surrey Activities Beyond LEO}

Anticipating the requirement for low cost access beyond LEO, Surrey has been actively working towards meeting this commencing with involvement on the STRV-1 missions from 1991 and the internally initiated and funded "Earthrise" lunar mission study in 1995 [6].

Over the past nine years Surrey has acquired flight heritage in the harsh radiation environment of GTO and has gained wide experience in Lunar and Interplanetary mission studies - both in-house and as contracts for ESA and NASA. Surrey power unit, batteries and payload have flown in GTO (STRVla,b,c,d) - and exceeded their design lifetimes. In-house studies have been performed on missions to the Moon, Venus, Mars, Near Earth Objects and specialised orbits such as LaGrange points. SSTL has performed phases B/C/D of the LUNARSAT mission study with the LUNARSAT team for ESA.

Several missions for the study of geospace have been performed, including a Cluster-Lite proposal to ESA and the Magnetospheric MultiScale (MMS) mission study for NASA. Surrey has also recently won a study contract for the ESA Rosetta comet lander momentum wheel. This stepwise evolution of the low cost planetary capability at Surrey is illustrated in Figure 3. 


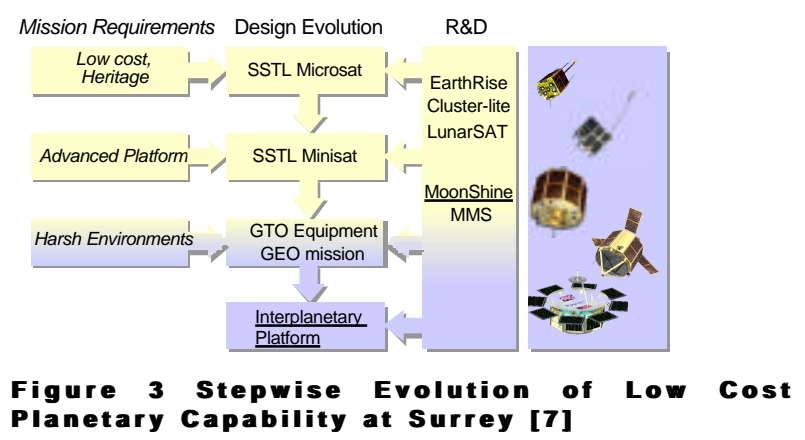

The Surrey Space Centre is a combined academic and commercial team comprising, SSTL (which carries out all commercial activities), and, CSER, a thriving academic research team. CSER offers many complementary activities which benefit SSTL's work on low-cost planetary mission analysis these include:

- space mission analysis

- payload development

- space environment research

- development of propulsion systems employing "safe" propellants (e.g. electrothermal resistojet, hybrid rocket motor, cold gas systems)

- astrodynamics R\&D (validation using planned and in-orbit Surrey Satellites)

* formation flying

* combined attitude and orbit control

* orbit and trajectory propagation

\section{Lunar Minisatellite \& Interplanetary Bus Missions: Mission Drivers}

For both the Lunar minisatellite, and the interplanetary platform the main mission drivers are:

- Low cost - to make the mission affordable on a national (or few nations) or commercial level

- No undue risks to be taken

- Repeatable, flexible missions to

* variety of lunar and interplanetary targets

* accommodate a variety of instruments and payloads

\section{General Mission Design Approach}

Surrey's approach to Low Cost Satellite Engineering and Small Satellite Programme Management will be employed to ensure that cost, risk and performance are effectively managed in order to achieve the targets for each mission. In addition, key features are use of :

- COTS components -which when carefully selected, can withstand total dose radiation of at least 10-15 krad (Si) (sometimes significantly more)
- functional (layered) redundancy - as employed on all Surrey modular microsatellites

- on-board and ground station autonomy

Choice of launch vehicle, trajectory and spacecraft propulsion are predominantly driven by mission cost. Repeatable, flexible missions impose an additional constraint - requiring both repeatable design choices and fully accountable costs.

The generic platforms will also provide mission flexibility through accommodation of a wide range of instruments and payloads. Payloads with special accommodation requirements will always be considered, but typically cannot drive the design, as in the case of high cost, traditional space missions. It may therefore be necessary to trade on requirements in order to meet both mission and spacecraft cost and design goals.

The next section of the paper focuses on key aspects of the modified minisatellite mission, MoonShine.

\section{MoonShine Mission Aims}

The key goal of the Moonshine mission is:

- to reach the Moon for $\$ 16-\$ 25 \mathrm{M}$

Meeting of this single objective would signify mission success.

There are, however, additional aims driven by Surrey's Lunar and Interplanetary Mission Mandate. These may be summarised as:

- to extend Surrey Mission capabilities beyond LEO

- to demonstrate Surrey subsystems and platforms in harsh, challenging environments

- to support a wide variety of missions to the Moon, NEOs and geospace orbits on a regular, reliable and affordable basis

- to accommodate a wide range of lunar and interplanetary payloads on low cost missions

In achieving these goals, MoonShine, should stimulate successive missions to the Moon, LaGrange points, Near Earth Objects and other unusual orbits on the modified minisatellite, ultimately increasing mission frequency and enabling frequent flight opportunities for science instruments and payloads.

\section{MoonShine: Mission Design}

\section{Study Requirements}

The study aim was to identify options for a mission to the Moon, based on Surrey's minisatellite platform. The first 
minisatellite, UoSAT-12 (Figure 4), was launched in April 1999. Four minisatellites carrying payloads for commercial agriculture applications are due for launch in 2002 and 2003.

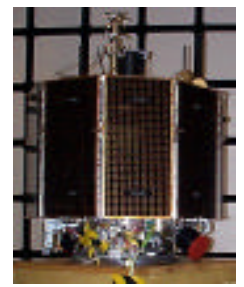

Figure 4 UoSAT-12 Minisatellite, launched 1999.

SSTL's minisatellite structure is qualified up to $400 \mathrm{~kg}$, the lunar mission total mass should therefore not exceed this value. Platform dimensions are $1100 \times 1100 \times 885 \mathrm{~mm}$, excluding external equipment.

Based on this standard minisatellite, different lunar mission designs are possible and obviously depend on the mission drivers. For example, cost and demonstration/validation of new technologies as drivers results in an experimental solution to the mission [8]. The identified options were assessed in terms of cost, performance, risk and degree of modification to the minisatellite - with low cost and demonstration of a repeatable capability as the main drivers - resulting in the MoonShine mission baseline presented in this paper.

\section{Launch}

Launch vehicle drop-off parameters determine the spacecraft orbit plane and Earth-Moon plane relative geometry. Suitable, transfer strategies to lunar orbit from this point have been identified by trading between several interdependent parameters - propellant use efficiency, trajectory inherent safety, time for transfer and associated radiation effects. Launches to Geostationary Transfer Orbit (GTO), direct lunar injection and intermediate orbits were considered.

Secondary launch opportunities into GTO for the $400 \mathrm{~kg}$ platform meet mission requirements through:

- regular launches to GTO orbits with fixed parameters

- low cost -- $\leq 12$ Million USD for Ariane launch to GTO [9]

Disadvantages that are critical to the design are:

- no control over the launch time -meaning that the mission design cannot assume favourable launch windows (to minimise delta-V) and the mission must be designed to cope with any GTO-LTO plane geometries

- GTO radiation environment

Direct and intermediate launches can eliminate both disadvantages encountered by GTO launch. They are typically dedicated and so launch date and the drop off parameters may be chosen to:
- $\quad$ significantly minimise the transfer delta- $\mathrm{V}$ requirements

- avoid the radiation belts (e.g. a 40, 000 x 400, $000 \mathrm{~km}$ intermediate orbit)

Direct injections requiring rapid spacecraft commissioning, quickly followed by critical lunar capture burn, are not suited to low cost operations. Launch to intermediate orbits offer a solution favourable in terms of radiation environment, low cost operations and delta- $\mathrm{V}$ - but still typically suffer from high costs, infrequent service associated with dedicated launches.

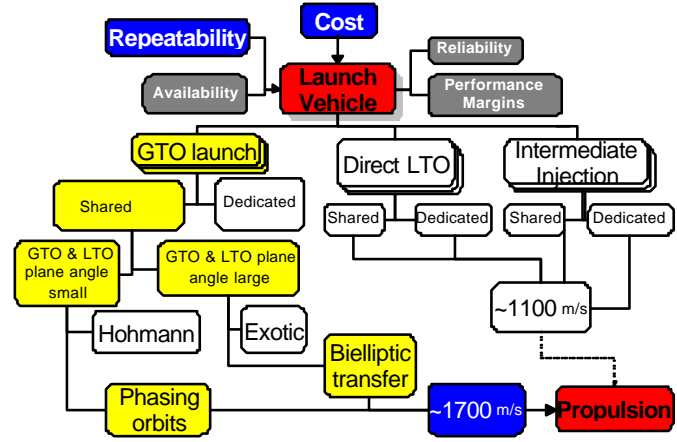

Figure 5 Launch-Driven Spacecraft Cost and Design Impacts: Decision Tree

It is worth noting that the increasingly competitive prices of several launch vehicles (Tsyklon and Dnepr, in particular) offering low-cost dedicated launches for small missions. However, until such launches are shown to be regularly available the mission baseline must assume a launch to GTO. Figure 5 shows the launch-driven spacecraft cost and design impacts. Surrey is currently negotiating on a number of such low-cost 'intermediate' launch opportunities and, if successful, these could be exploited to derive additional payload performance, extended mission lifetime, or cost benefits over the baseline mission from GTO.

The baseline spacecraft propulsion system should provide a $1700 \mathrm{~m} / \mathrm{s}$ velocity increment to cover transfer to Lunar encounter from any GTO launch, Lunar orbit capture and a one year mission in a low lunar orbit (Table 1).

\begin{tabular}{|l|c|}
\hline Propullsive mamoeuwre & moom \\
\hline Earth Departure & 690 \\
\hline Mid-course Corrections & 100 \\
\hline Injection Burn & 810 \\
\hline Orbit Maintenance \& ADCS (1 Year) & 100 \\
\hline Total Delta-V (m/s) & 1700 \\
\hline
\end{tabular}

Table 1 Lunar Mission Delta-v Requirements

\section{Trajectory}

The proposed trajectories illustrated in Figure 6 and Figure 7 both employ phasing orbits (segmented Hohmann transfers) out of GTO to lunar encounter. If large plane angles between GTO and LTO are encountered bielliptic or weak stability boundary transfers could be employed to avoid high 
propellant plane change manoeuvres. These safe, low-cost transfer strategies from GTO have been analysed in detail in the LUNARSAT phase B/C/D study, performed by SSTL and the LUNARSAT team for ESA [10] - and have been shown to satisfy all GTO-LTO geometries.

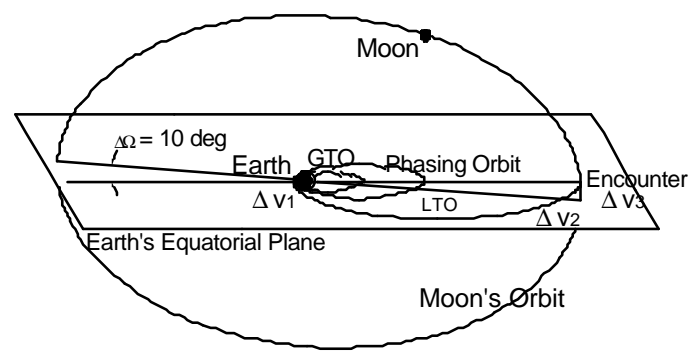

Figure 6 Phasing Orbits to Lunar Encounter for Small LTO-GTO PIane Angles

As part of the transfer strategy, less than one month can be tolerated in the GTO radiation environment. In the worst GTO launch case, it can be a few months before the correct geometry is presented. To overcome this small burns will be applied in GTO, as necessary, to move out of the most severe environments before the total acceptable radiation dose is approached. The additional delta- $\mathrm{V}$ can be met though trajectory optimisation and mission margins or in the worst case, by trading on mission lifetime.

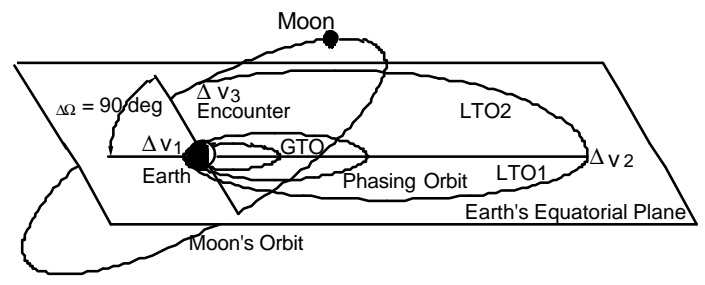

Figure 7 Phasing Orbits and Bielliptic Transfer for Large GTO-LTO Plane Angles

The use of phasing orbits approach is, additionally, well suited to low-cost autonomous operations approach - breaking the major burns down into smaller velocity increments, and allowing ample time for spacecraft commissioning and orbit determination before and after propulsive burns.

\section{Space Segment}

\section{Propulsion}

The propulsion system requirements are:

- compatibility with the Surrey minisatellite

- to deliver up to $1700 \mathrm{~m} / \mathrm{s}$ velocity increment

\section{Minisatellite Propulsion System Accommodation}

One option considered is a "bolt-on" propulsion unit to the standard minisatellite (Figure 8). Four commercially available spherical tanks hold approximately 180 litres of propellant and it may be possible to increase this volume should other suitable tank configurations be identified. High performance propulsion systems will be required to provide the required delta- $\mathrm{V}$ in the available volume.

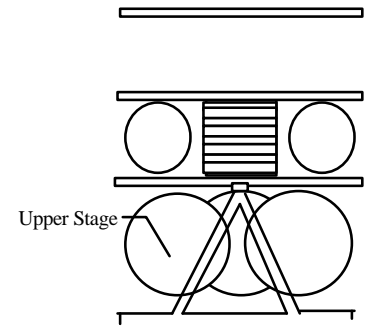

Figure 8 Standard Minisatellite with Bolt-on Propulsion Unit: Option 1

This option is excellent in terms of assembly integration and test (AIT) and offers a neat modular approach to the propulsion system. The drawback is that a second propulsion system, capable of holding roughly $100 \mathrm{~m} / \mathrm{s}$ delta-V, would be required within the minisatellite for ADCS and orbit maintenance.

The second considered configuration is the minisatellite with a built-in propulsion system - this is a volume-limited solution and high performance propulsion systems will be required. All propulsion tanks, pipework and load-bearing structure are approximately contained in bottom of $\mathrm{s} / \mathrm{c}$ which eases AIT. Roughly 180 litres of propellant can be held in three spherical and three long cylindrical tanks (the top view is like a standard minisatellite) as shown in Figure 9.
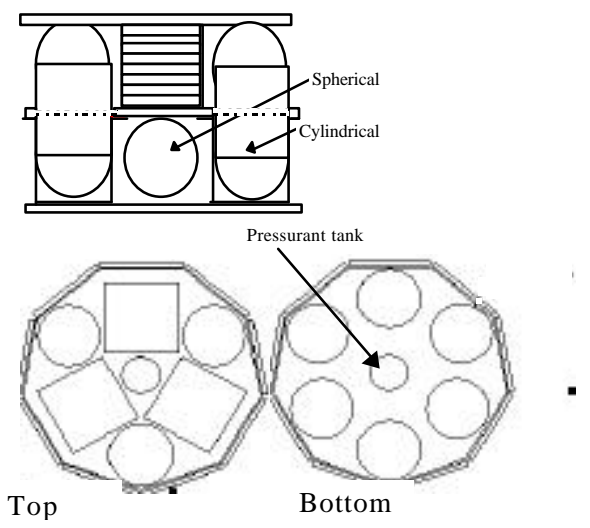

Figure 9 Modified Minisatellite with Built-In Propulsion Capability: Option 2

The third considered configuration option can offer approximately 300 litres propellant storage, employing, in this case, three long cylindrical tanks (Figure 10). A thrust tube around the tanks and pipework would be used to bear the loads to the attach fitting and isolate the propulsion system from electronics. Spacecraft subsystems, developed for the inorbit SNAP nanosatellite, are contained in 168x122x20.7 mm 
size modules (slightly bigger than Eurocard size PCB's) and may be mounted to the outside thrust tube.

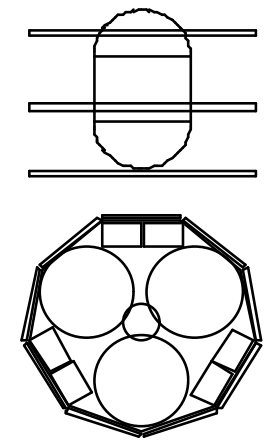

Figure 10 Modified Minisatellite with Built-In Propulsion Capability: Option 3

This option allows flight of lower (recurring) cost, lower performance propulsion systems - or a significantly increased delta-V capability with high performance systems - enabling a wide range of traditional and experimental propulsive technologies could be accommodated. This option was not considered further as the modifications to the minisatellite were the greatest. However, now that the small "SNAP" subsystems have been developed and flown option 3 will be reconsidered. The aim will be to establish cost-performancerisk differences compared to option 2 and a "clean sheet" mission design.

\section{Propulsion System Selection}

Traditional and in-house bipropellant, monopropellant and hybrid propulsion options, considered for the mission are listed in Figure 11. These were assessed relative to each other in terms of performance, estimated recurring engineering cost and technical risk.

\begin{tabular}{|l|l|l|}
\hline Propulsion & \multicolumn{1}{|l|}{$\begin{array}{l}\text { Planetary } \\
\text { Heritage }\end{array}$} & Comment \\
\hline Bipropellant & Yes & $\begin{array}{l}\text { Traditional solution: high } \\
\text { performance, high recurring cost; } \\
\text { low technical risk }\end{array}$ \\
\hline $\begin{array}{l}\text { NTO \& } \\
\text { MMH }\end{array}$ & No & $\begin{array}{l}\text { In-house study: good performance, } \\
\text { lower recurring cost after R\&D; } \\
\text { high technical risk }\end{array}$ \\
\hline $\begin{array}{l}\text { HTP \& } \\
\text { kerosene }\end{array}$ & $\begin{array}{l}\text { In-house study: poor volume } \\
\text { performance, very low recurring } \\
\text { cost after R\&D; modest technical } \\
\text { risk }\end{array}$ \\
\hline $\begin{array}{l}\text { Nitrous \& } \\
\text { kerosene }\end{array}$ & No & $\begin{array}{l}\text { Traditional solution: modest } \\
\text { performance; modest recurring cost; } \\
\text { modest technical risk }\end{array}$ \\
\hline Monopropellant & Yes & $\begin{array}{l}\text { In-house study: good performance; } \\
\text { low recurring cost; modest } \\
\text { technical risk }\end{array}$ \\
\hline Hydrazine & $\begin{array}{l}\text { In-house development: good } \\
\text { performance; low recurring cost; } \\
\text { modest technical risk }\end{array}$ \\
\hline HTP & No & In house development: good \\
\hline Hybrid & No &
\end{tabular}

\begin{tabular}{|l|l|l|}
\hline & & $\begin{array}{l}\text { performance; low-med. recurring } \\
\text { cost; high technical risk }\end{array}$ \\
\hline $\begin{array}{l}\text { Nitrous \& } \\
\text { PE or HTBP }\end{array}$ & No & $\begin{array}{l}\text { In house study: good performance; } \\
\text { low recurring cost; med-high } \\
\text { technical risk }\end{array}$ \\
\hline
\end{tabular}

Figure 11 Modified Minisatellite: Moonshine Propulsion Options

Figure 12 compares the performance of the options in terms of propellant mass and volume for a $1700 \mathrm{~m} / \mathrm{s}$ velocity increment. Approximate volume and mass limits are indicated, based on the volume-limited options 1 and 2 and a $400 \mathrm{~kg}$ spacecraft mass. Compatible options in terms of performance and minisatellite accommodation are therefore:

- Hydrogen Test Peroxide (HTP) and Polyethylene (PE) hybrid

- Nitrogen Tetroxide (NTO) and Mono-Methyl Hydrazine (MMH) bipropellant (or NTO \& Hydrazine)

- HTP \& Kerosene bipropellant (or similar)

- HTP monopropellant (marginal for mass performance)

Trajectory optimisation should also offer increased compatibility options - but at this stage in the design margins will be kept.

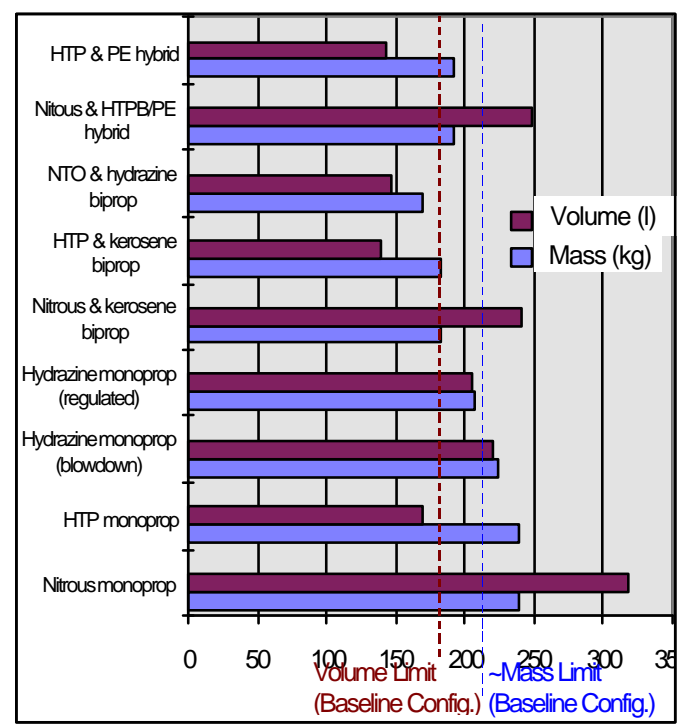

Figure 12 Spacecraft Propulion Options: Propellant Mass and Volume Performance Comparison

Three of the compatible propulsion options are in-house proposals. The SSC propulsion goals are to develop technologies that provide viable, cost effective propulsion solutions, for small spacecraft applications. Environmental, logistical (export, shipping and launch preparation) and safety aspects of SSC propulsion, throughout the mission lifecycle, are recognised as cost drivers [11]. Hence, Surrey avoids use of dangerous and environmentally damaging fuels and oxidisers 
- and all propulsion research is conducted in a university environment.

To-date, Surrey has successfully demonstrated cold gas and electrothermal resistojet propulsion technologies in orbit - but these have only been required to deliver small velocity increments. Until Surrey has demonstrated higher delta-V systems the technical risk for in-house solutions will obviously be very high and run the risk of becoming schedule drivers. More mature than Surrey's bipropellant R\&D - the hybrid development programme at Surrey is currently working towards validation of $\sim 300 \mathrm{~m} / \mathrm{s}$ hybrid rocket motor on a lowcost mission. Until then, given the cost-risk-performance matrix, MoonShine will baseline a COTS bipropellant system. (An experimental approach to MoonShine would result in a monopropellant option - less technical risk than a hybrid, but simpler and lower cost the bipropellant options.)

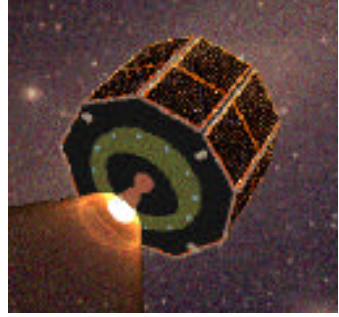

Figure 13 Moonshine Propulsion Concept

A single main engine, providing between 50 and $200 \mathrm{~N}$ thrust,. will be mounted aligned to the spacecraft $\mathrm{Z}$ (thrust) axis and directed through centre of gravity (Figure 13).

Electric propulsion options were also considered - as their typically high specific impulses (Isp) offer significant volume and mass savings. The drawbacks are long transfer times, associated with the tiny levels of thrust, and large power requirements. Higher power configuration options are possible with the minisatellite - these could bring transfer times down to between 12 to 24 months. At this stage, thermal, radiation and other areas of electric propulsion impact have not been fully considered. Preliminary indications are, however, that building the platform around the propulsion system in a more "traditional" approach would be better suited to an electric propulsion system.

\section{Orbit Determination}

Orbit determination is a key challenge for any planetary mission, especially at low cost. Two phases of orbit determination are identified:

- Earth-centred orbit determination of the transfer orbit

- Earth- or Moon-centred orbit determination for nominal lunar operations

and both must be based on autonomous, low-cost ground station (s) and a small operations team.
Transfer orbit determination options considered were:

- Norad Radar tracking for Keplerian elements

- Traditional ranging using retransmission of ranging tones through the telemetry system

- Use of the Deep Space Network (DSN)

- Coded pulse ranging using retransmission of a coded pulse through the telemetry system

- Ground based GPS-like transmissions from network of Surrey Ground Stations (GSNs) to the spacecraft

- Transmission of GPS-like signal from the spacecraft to a network of three or more Surrey GSNs

Trading between cost, performance and compatibility with existing Surrey infrastructure has led to a coded pulse S-band ranging baseline. This ranging method has been demonstrated on the AMSAT Phase 3C mission [12]. No additional hardware is required on the spacecraft (the GSN transmits a continuous pulse, which the spacecraft re-transmits) and minimal hardware is needed at the GSN, although development of the GSN software will be necessary.

Moon-centred lunar orbit determination may be performed with a radar altimeter. Surrey is currently investigating the use of the spacecraft S-band communications system for this purpose to enable accurate, fully autonomous lunar orbit determination. The altimeter frequency could also be selected to enable it to operate as a ground penetrating radar payload that could provide information on the subsurface structure and presence of water ice.

Earth-centred orbit determination for a spacecraft in lunar orbit may be sufficient, depending on orbit height above lunar surface and accuracy of spacecraft orbit knowledge required data for payloads. One accurate method is GPS-Like Tracking (GLT) [12]. It is proposed that a GLT experiment - already under development at Surrey - is flown and tested on the MoonShine mission. This would offer functional redundancy with the S-band ranging system, but must not be allowed to drive up mission costs (ie requirement for more than one GSN).

GLT would incorporate a transmitting beacon on the satellite whose signal structure can be tracked in GPS receivers located at the ground stations. The ground station receivers should be able to track both GPS (to provide accurate time synchronisation of the stations) and non-GPS spacecraft (MoonShine). 


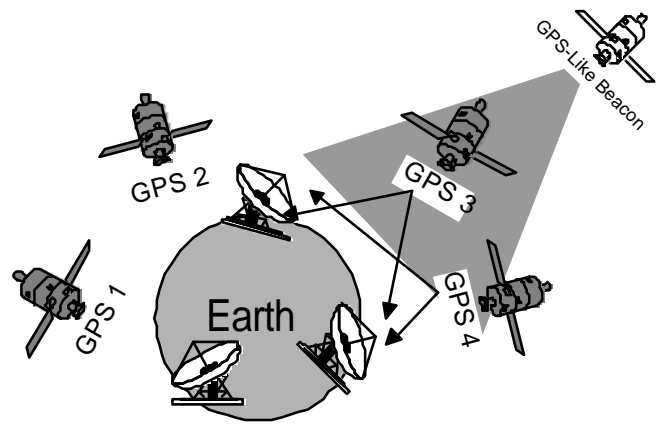

Figure 14 GPS-Like Tracking Concept

Building a GLT receiver entails some modification to Surrey's existing space qualified GPS receiver [14]. The GLT would need to transmit at relatively high power for a few minutes at a time (depending on how long it takes to get a fix). The available on-board power and the required determination accuracy will determine the number of fixes. In GLT the carrier phase from the MoonShine spacecraft is included as with differential GPS and the orbit is estimated in the same way.

\section{Subsystems Overview}

An overview of the remaining MoonShine subsystems follows. There are areas of commonality between some MoonShine and Interplanetary Platform subsystems or modes of operation and these will be covered together in the description of the Interplanetary platform.

\section{ADCS}

In lunar orbit the spacecraft will be three-axis zero momentum stabilised using four reaction wheels mounted in a tetrahedron and providing control accuracy to $0.1^{\circ}$ in the pitch, roll and yaw. This configuration will ensure that the various conflicting pointing requirements are met for payload operations (nominally Moon pointing), communications (Earth pointing), thermal and power purposes. Momentum bias mode offering high platform stability is also possible, if desired. Four, small, thrusters will be used and to de-saturate the reaction wheels and to spin up and spin down the spacecraft, which will be spin stabilised during all major propulsive manoeuvres.

Gyros and accelerometers are employed for spin rate and axis determination during thrusts. Four sun sensors and two star cameras provide attitude knowledge. All proposed attitude modes have been demonstrated by UoSAT-12. The sensors and actuators (with the exception of the thrusters) are all Surrey-built and have been demonstrated in orbit (Figure 15) offering good performance, and a flexible attitude solution at low cost.

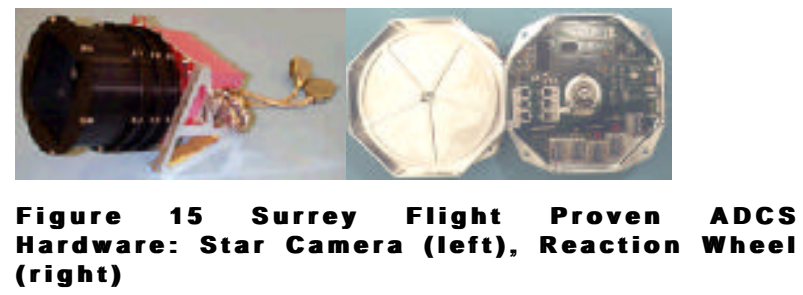

\section{Power}

Power is provided by eight body mounted GaAS solar panels, three $7 \mathrm{Ahr} \mathrm{NiCd}$ battery packs, and a centralised power switching system distributes power from the $28 \mathrm{~V}$ unregulated bus. The batteries provide storage power for peak demand or to survive several hours in eclipse. The proposed solution generates over 100 Watts during transfer and in lunar orbit, leaving more than $30 \mathrm{~W}$ for the payloads.

The power subsystem is not optimised and it may yet be desirable to trade on factors such as further reducing costs, increasing power or reducing mass - potentially leading to use of silicon panels, higher energy density batteries and lightweight technologies - as in the solution for the Interplanetary Platform. Additionally, it may be necessary to deploy some of the body mounted panels or mount a string of solar cells to the $+Z$ and $-Z$ facets to ensure sufficient power for recovery during attitude anomalies.

\section{Communications}

The communications system employs dual redundant S-band receiver and transmitter (which is configured into a ranging transponder for orbit determination) with, a 3.5 metre ground station dish and an array of patch antennas on one spacecraft side panel (in place of ninth solar panel). A 4W RF power downlink uses a Viterbi coding scheme to support a $10 \mathrm{kbps}$ downlink with a $3 \mathrm{~dB}$ link margin.

A 1 kbps uplink is available for spacecraft commanding and an omni-directional patch antenna on the $+\mathrm{Z}$ and $-\mathrm{Z}$ facets will allow commanding in all attitude modes.

\section{Command and Data Handling}

The command and data handling subsystem consists of two SSTL 80386-based on board computers (OBC), Ethenet links and a triple redundant Controlled Area Network (CAN) bus. The Ethernet and CAN handle spacecraft internal communications, while the OBCs, each with 256 Mbytes of SRAM, handle satellite control and housekeeping functions; capture, process and format payload data; and manage the communications link. data A 1 GByte DRAM Solid State Data Recorder (SSDR) allows point-to-point data capture up to 80 Mbps direct from payloads to downlink. 


\section{Environmental}

Thermal surfaces and overall spacecraft heat bias will provide passive thermal control. Each propellant tank will have its own heater, operating in fail safe mode. The batteries may also require heaters for long eclipse periods.

For a 30 day wait in GTO, a few months phasing to lunar capture and a year in lunar orbit, roughly $13.5 \mathrm{krad}(\mathrm{Si})$ dose is expected for a $2 \mathrm{~mm}$ aluminium structure (assuming also that small burns can be performed to avoid the most severe environments). To reduce the encountered dose spot shielding of sensitive components that must be on can be employed, and any reduction in the length of time in GTO is significant.

\section{Payload Mass and Accommodation}

MoonShine can support $20 \mathrm{~kg}$ payload into low circular lunar orbit from the baseline GTO mission. The spacecraft mass breakdown is shown in Figure 16. For illustration purposes - a $500 \mathrm{~m} / \mathrm{s}$ reduction in delta- $\mathrm{v}$ which would readily be achieved from an intermediate launch can support over $70 \mathrm{~kg}$ payload. These values represent a spacecraft design that has not undergone any mass optimisation, so trading between cost and optimisation may yet see additional increases in payload performance to lunar orbit.
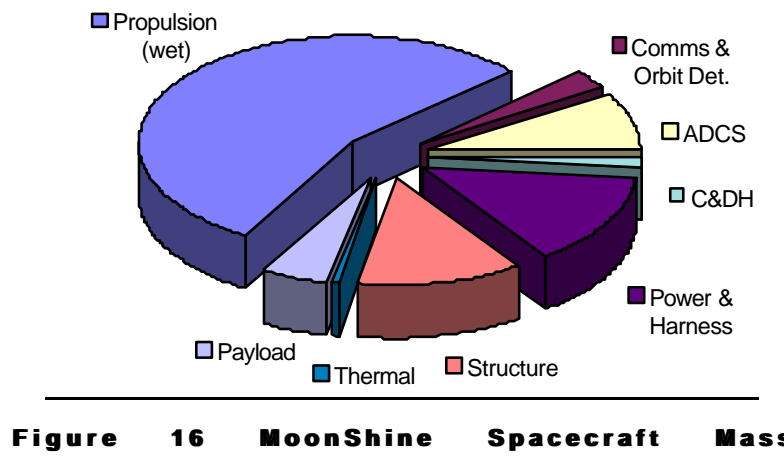
Breakdown for Missions from GTO
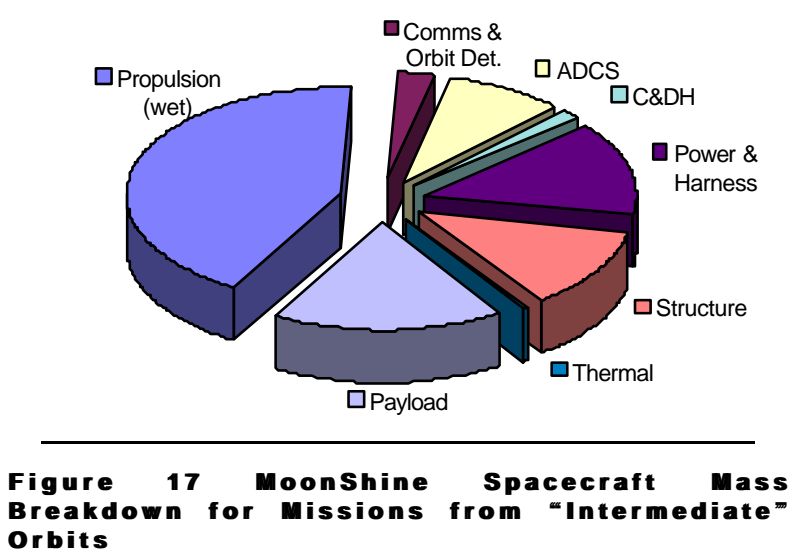

Payloads may be housed in an internal payload stack and module trays in the two platform stacks. Additionally the external frame can support telescopes, antennas, sensors and other payloads (Figure 18).

Mission operations, autonomy and risk strategies; Mission applications; and Programmatics are covered for both platforms - after the following overview of the Interplanetary Platform.

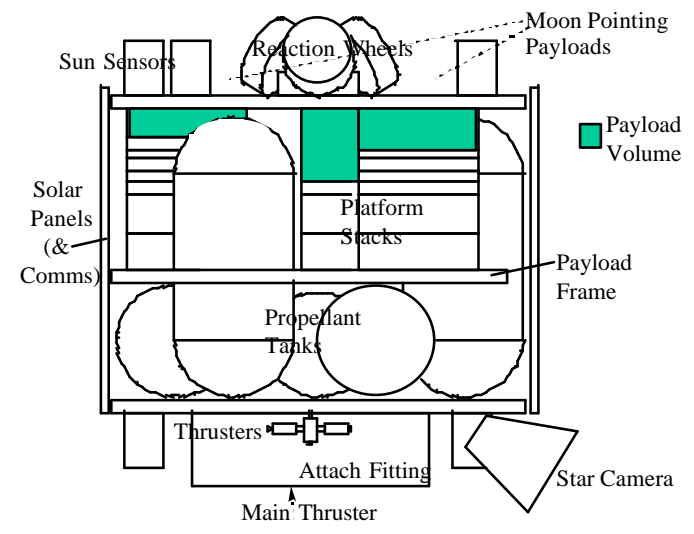

Figure 18 Moonshine Internal Configuration

\section{Interplanetary Platform [15]}

The objectives of the Interplanetary Platform (IPP) study were to:

- capitalise on the lunar mission studies

- determine the drivers for an inner planets mission.

Most importantly, it was to determine how mission risk could be significantly reduced to avoid it becoming a cost driver.

The key mission driver for a Mars and Venus mission has been identified as the communications system, and for a Mercury mission, radiation and the thermal environment become significant drivers, along with an extremely challenging delta-v requirement. Missions to Mars and Venus using the IPP are described.

\section{Launch and trajectory}

Launch is the predominant cost and design driver, and for the purposes of this study, launches into LEO, GTO and Geostationary Orbit (GEO) were considered. As with the modified minisatellite platform, a number of low cost launch offers have been identified, but are precluded from further consideration as they are not easily demonstrated as being repeatable.

After assessment of the repeatable launch vehicle options a dedicated Athena-2 (LMLV-2) launch vehicle was baselined, capable of injecting around $590 \mathrm{~kg}$ into GTO, for an approximate cost of $\$ 32$ million (FY2003). This US launch 
vehicle successfully launched the low cost Lunar Prospector mission in early 1998.

The delta- $\mathrm{v}$ requirements for several possible targets were assessed considering only direct transfers, and these appear in Table 2. Initial encounter orbits were optimised for minimum risk. Successive firings of the propulsion system would reduce the periapsis to the desired operating orbit.

\begin{tabular}{|l|l|l|l|}
\hline $\begin{array}{l}\text { Propulsive } \\
\text { Manoeuvre }\end{array}$ & Mercury & Venus & Mars \\
\hline Earth Departure & 2,964 & 1,040 & 1,156 \\
\hline Plane Change & 1,343 & 799 & 517 \\
\hline $\begin{array}{l}\text { Mid-course } \\
\text { Correction (est.) }\end{array}$ & 100 & 100 & 100 \\
\hline Injection Burn & 2,855 & 467 & 702 \\
\hline $\begin{array}{l}\text { Orbit Maintenance } \\
\text { \& Margin (1 Year) }\end{array}$ & 746 & 260 & 267 \\
\hline $\begin{array}{l}\text { Total Delta-V } \\
\text { (m/s) }\end{array}$ & 8,008 & 2,666 & 2,742 \\
\hline
\end{tabular}

Table 2 Interplanetary Platform Delta-v Requirements

\section{Propulsion and AODCS}

The spacecraft is spin stabilised to $10 \mathrm{rpm}$ during engine firing and to $1 \mathrm{rpm}$ for all interplanetary ballistic phases. In orbit, the spacecraft remains spin stabilised with the antenna pointing to Earth, although there is the potential to configure for momentum bias mode about the major spin axis depending on the exact complement of payloads. The attitude Control System employs spin up and spin-axis precession thrusters, and two momentum wheels. Three-axis quartz gyros are employed for spin rate and axis determination, and a pair of star cameras provides $0.1^{\circ}$ attitude knowledge.

As a result of the high delta-v requirements, a COTS bipropellant system was baselined. To minimise system cost, MMH and Nitrogen Tetroxide (NTO) was selected - requiring 4 identical tanks. The system was sized for a delta-v requirement of $3200 \mathrm{~ms}-1$. A separate high- pressure nitrogen tank pressurises the MMH and NTO teardrop shaped tanks, and acts as propellant for the cold gas thrusters. A single 100 $\mathrm{N}$ thruster was baselined for the main engine burns, typically requiring 100 orbits while in GTO for the interplanetary injection, and approximately 34-70 minutes of continuous thrusting for the target planet orbit insertion burn.

Dual mode star cameras are employed to measure the sun diameter in order to offer an estimate of distance to the spacecraft This estimate is then used to determine the direction of Earth. A hardware mission clock is employed for ambiguity resolution in autonomous orbit determination when the spacecraft has reached Mars or Venus orbit. For more precise ground assisted navigation, an S-band ranging transponder is carried, and the spacecraft omni antennas ensure the operators can assist the spacecraft with acquisition

\section{Communications}

The communications system employs an S-band ranging transponder with dual redundant receivers, a 7.3 metre ground station dish and a $1.9 \mathrm{~m}$ spacecraft dish. Conventional protocols and coding are employed in order to allow COTS equipment to be used on the ground. The uplink can support a command rate of $100 \mathrm{bps}$ at the maximum distance of 380 million km with sufficient operating margin. Command to the spacecraft low gain antenna is impractical using this set-up and would require higher EIRP's to sustain the link. The 20W RF power downlink uses Viterbi and RS coding schemes supporting a downlink of $8 \mathrm{bps}$ at the maximum distance, resulting in $1 \mathrm{Mbit} /$ day of mission data.

\section{Power}

Silicon panels have been selected for reasons of cost, and in order to limit mass two lithium ion battery packs providing 140WHr of storage power for eclipses or peak power demand are included. A centralised power switching system distributes power from the $28 \mathrm{~V}$ unregulated bus.

Adequately sized solar arrays on all facets permits a simple autonomous power safe mode. A simple search for the sun is initiated using a coarse sun sensor, after a star camera fix determines the ecliptic plane. SSTL's LEO satellites reduce power to non-critical subsystems in the event of a negative power budget and so automatic survival behaviour is standard practice. Majority voting on-board computers and a watchdog timer safeguards consumption of consumables. For a mission to Mars, the proposed solution generates more than 150 watts post processed power during normal attitudes, leaving more than $40 \mathrm{~W}$ for the payloads.

\section{OBDH and Software}

The on-board software will reside in two Intel-186 based computers, each with full Error Detection and Correction (EDAC), both will operating in a hot redundant mode. An additional 'watchdog' microcontroller facilitates majority voting for critical decisions, bulk data storage of payload data residing on a single space qualified hard disk. Internal communications will be provided by a dual Controlled Area Network (CAN) bus system.

\section{Environment}

The spacecraft will encounter an extremely hostile thermal environment during the various phases of the mission. An Earth facing spinner is not ideal since it will maintain the antenna pointing within 30 degrees of the Sun, the rest of the spacecraft will become a large space facing radiator, but MLI 
and active heaters ensure a suitable environment for the spacecraft.

Based on fifteen days in GTO, 100 phasing orbits, a 5-9 month cruise phase (Venus and Mars) and a 24 months operation phase the received dose is $\sim 13 \mathrm{krad}(\mathrm{Si})$. As with the Lunar mission, the predominant radiation driver is the time spent in GTO and again spot shielding of sensitive components that must be on is employed.

\section{Spacecraft Configuration and Payload Accommodation}

The spacecraft configuration requirements are a trade between the conflicting power, communications and science demands. The proposed solution for the Interplanetary Platform is a spinner with an Earth facing antenna facet (Figure 19). The platform system electronics are contained within a single module stack surrounded by 4 propellant tanks and a single nitrogen pressurant tank. Primary structure couples the subsystem and propulsion mass to the launch vehicle interface.

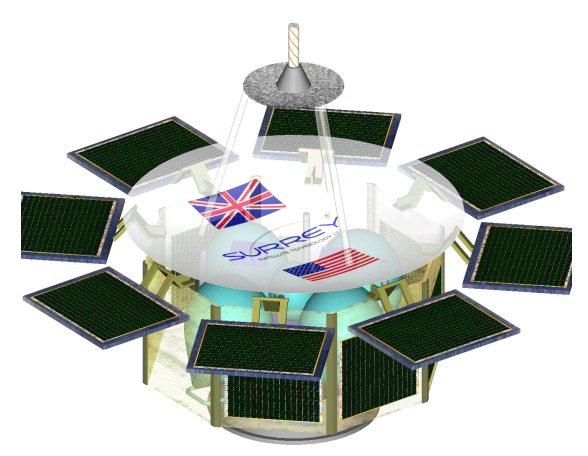

Figure 19 Proposed Interplanetary Mission Configuration

The mass budget is for a $576 \mathrm{~kg}$ spacecraft including $10 \%$ margin. A wide variety of science instruments, with a total mass up to $20 \mathrm{~kg}$, can be accommodated either on the antiEarth facet or radial facets.

\section{Lunar and Interplanetary Platform Missions}

\section{Operations, Autonomy and Mission Risk}

A major cost driver for traditional interplanetary missions is the operations concept. The Surrey operations strategy is based upon autonomous operations with operator intervention only for faults, this reduces cost and increases reliability.

The ground stations will consist of 3.5 and 7.3 metre S-Band tracking dishes in a protective radome, for the Lunar and Interplanetary Platform Missions respectively. Five computers will support operations - including platform analysis, control and data entry and archiving. Payload data will be sent to the user via the Internet.

Unlike low cost LEO missions, critical orbit injection manoeuvres - such as GTO perigee burn and orbit insertion burn - must be undertaken and these are likely to be scheduled while outside of direct ground station line-of-sight communications. The problems are compounded by one way signal delays (up to 20 minutes for the considered interplanetary missions - although this problem is not dissimilar to typical low cost LEO missions where 2-hour revisit times are typical). Consequently, an on-board autonomous propulsion controller must operate. SSTL, in conjunction with Microcosm Inc. already has experience of this with the Autonomous On-board Control Kit (OCK) software. UoSAT-12 has been 'flying' autonomously since shortly after launch, undertaking minor orbit corrections for some 6 months without user intervention. It is proposed that a derivative of this software is utilised for propulsive manoeuvres.

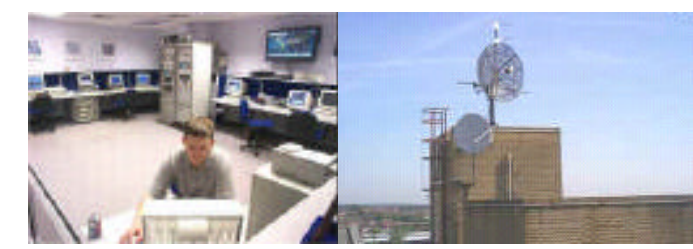

Figure 20 Surrey Mission Control Centre (left) Surrey Tracking Antenna (right)

Technical risk has been minimised throughout all phases of the mission designs. Tolerant interplanetary injection trajectories are proposed in which each individual propulsive burn at GTO perigee is not critical (e.g. $10 \mathrm{~ms}-1$ velocity increments proposed on the Mission to Mars). After each burn the orbit is characterised. Planetary injection burns will be handled by the autonomous propulsion controller. Power and communications are designed to cope in uncontrolled attitude modes.

\section{Mission Applications}

Traditionally, missions proposed identify science requirements and then develop an instrument suite and platform to meet these objectives. A converse design-to-cost approach has been followed here, resulting in two low cost platforms that can accommodate a variety of payloads on a range of lunar and interplanetary missions.

Some of the missions studied at the Space Centre, that can be performed by the proposed platforms are:

- Lunar Orbiter

- Lunar Impactor 
- Mars Orbiter

- Venus Orbiter

- Near Earth Asteroid Mission

- Missions to LaGrange Points

- Magnetospheric Missions \& "Space Weather" (other Surrey platforms are also suitable for these missions)

Surrey remains open to mission ideas and is seeking support from partners and Principle Investigators - on new or proposed missions. The aim is to thoroughly identify science requirements and instruments to fulfil valuable scientific goals using the modified minisatellite and interplanetary platforms.

\section{Programmatics}

The development schedule for both missions is 3 years duration from initial concept to launch, with initial launch readiness possible by mid 2003 .

Costs established from Surrey's various lunar mission studies have ranged from $\$ 16-\$ 25 \mathrm{M}$. Selecting a repeatable Ariane launch to GTO at $\$ 12 \mathrm{M}$ places the Moonshine current baseline between $\$ 20-25 \mathrm{M}$ - at upper end of this spectrum. The estimated cost of a repeatable mission concept for the Interplanetary Platform using the Athena-2 Launch vehicle is presented in Figure 7. These costs include launch, operations and typical payload related costs.

\begin{tabular}{|l|c|}
\hline & Cost (Million \$) \\
\hline Platform & $14,200,000$ \\
\hline Groundstation \& Operations & $3,580,000$ \\
\hline Launch and Support & $32,500,000$ \\
\hline TOTAL & $\mathbf{5 0 , 2 8 0 , 0 0 0}$ \\
\hline
\end{tabular}

Table 3 Interplanetary Platform Mission to

Mars Project Cost Breakdown

In both designs overall mission cost is dominated by the cost of a choice of repeatable launches. Further (significant) cost reduction can be achieved by opting for a launch from the Commonwealth of Independent States (CIS), which Surrey has considerable experience managing. A CIS repeatable mission to Mars or Venus, using the interplanetary platform could be undertaken for around $\$ 37$ million, and a mission to the Moon for less than $\$ 20$ million.

\section{Conclusions}

The low cost lunar mission will validate a repeatable platform design, to meet the growing global requirement for affordable missions to the Moon, LaGrange Points, and other interplanetary trajectories. Between 20 and $70 \mathrm{~kg}$ or payload mass can be accommodated in lunar orbit. Missions to Mars, Venus and near Earth objects can be met by the SSTL Interplanetary Platform, carrying up to $20 \mathrm{~kg}$ of payload.
Together, the platforms will enable regular flight of payloads to the Moon and interplanetary space at unprecedented low cost.

Work continues at Surrey on the mission studies - looking in more detail at 'safe' trajectories, autonomy -and ways to further minimise cost and risk. As a commercial company, SSTL cannot raise all the funds necessary for Lunar and Interplanetary missions internally, and is seeking support from partners or Principle Investigators.

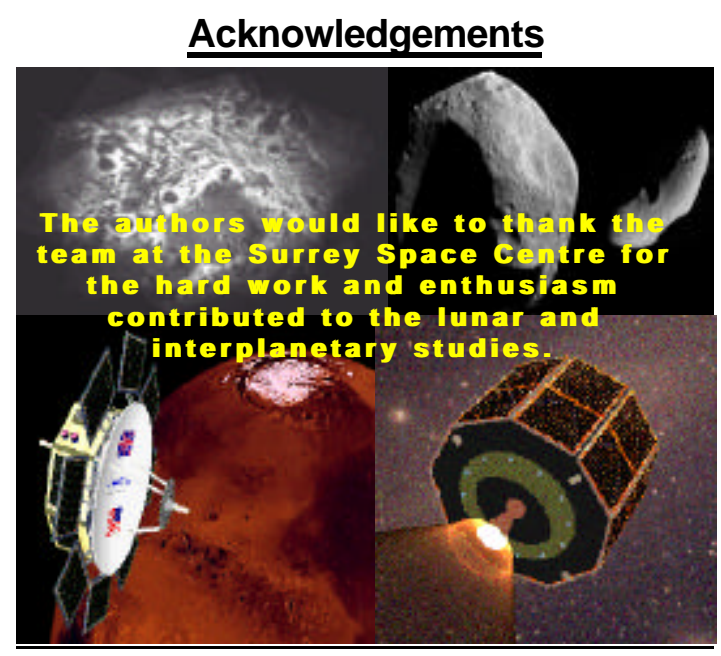

\section{References}

[1] Ward J et al "First in-orbit results of the UoSAT-12 mission" SSC99-I-2, 13 ${ }^{\text {th }}$ AIAA USU Conference on Small Satellites, Logan Utah, USA 1999

[2] Zheng Y., Sweeting Prof. M., "Initial Mission Status Analysis of 3-Axis Stable Tsinghua-1 Microsatellite" $14^{\text {th }}$ Annual AIAA/USU Conference on Small Satellites, Logan, Utah, August 1999

[3] Lancaster R., Underwood Dr. C., "The SNAP-1 Machine Vision System" $14^{\text {th }}$ Annual AIAA/USU Conference on Small Satellites, Logan, Utah, August 1999

[4] Ward, Dr. J., Jason S., Sweeting Prof. M., "Microsatellite Constellation for Disaster Monitoring" $13^{\text {th }}$ Annual AIAA/USU Conference on Small Satellites, Logan, Utah, August 1999

[5] Da Silva Curiel, R.A. and Jolly, G. "The GANDER Constellation for Maritime Disaster Mitigation", IAA-98C.1.02 
[6] Sweeting Prof. M., Curiel ADS, Monokosso N. "Low Cost Lunar Mission for the year 2000” IAF Beijing 1996

[7] Curiel R A da Silva, Phipps A., Jason S., Ward J W. "LunarSat and MoonShine: Small Satellites for Interplanetary Exploration" $22^{\text {nd }}$ International Symposium on Space Technology and Science, Japan 2000

[8] Sweeting Prof. M., Jason S., Ward Dr J., Curiel ADS, Phipps A., "MoonShine - A low cost Minisatellite Mission to the Moon" $2^{\text {nd }}$ Meeting of the Surrey Space Club, Singapore, Dec 1999

[9] Information provided by Jim Keravala, SSTL Launch Services Manager

[10] Lunar Academic Research Satellite LUNARSAT Phase C/D. Management and Commercial Proposal to ESA $19^{\text {th }}$ Feb 1999

[11] Haag G., Sweeting Prof. M., Richardson Dr. G., "Low Cost Propulsion Development for Small Satellites at the Surrey Space Centre" $13^{\text {th }}$ Annual AIAA/USU Conference on Small Satellites, Logan, Utah, August 1999

[12] AMSAT Website: http://www.amsat.org/. "Phase 3C" Mission

[13] Lichten S M, Sweeney D et al "New Ground and SpaceBased GPS Tracking Techniques for High-Earth and Deep Space Orbit Determination Applications" JPL

[14] SSTL SGR Data Sheet http://www.sstl.co.uk/.

[15] Sweeting Prof. M., Curiel ADS, Gomes L, Ward Dr J., "The Moon and Inner Planets - Getting there for Low Cost" $4^{\text {th }}$ AIAA Conference of Low Cost Planetary Missions, JHU/APL Washington May 2000 